\title{
Tecnologias em saúde e suas contribuições para a promoção do aleitamento materno: revisão integrativa da literatura
}

\author{
Health Technologies and their contributions to the promotion \\ of breastfeeding: an integrative review of the literature
}

Naélia Vidal de Negreiros da Silva ${ }^{1}$

Cleide Maria Pontes ${ }^{1}$

Nayara Francisca Cabral de Sousa ${ }^{1}$

Maria Gorete Lucena de Vasconcelos ${ }^{1}$

${ }^{1}$ Departamento de Enfermagem, Centro

de Ciências da Saúde, Universidade Federal de Pernambuco. R. Prof. Moraes Rêgo S/N, Cidade Universitária. 50670-901 Recife PE Brasil.

naeliav@yahoo.com.br

\begin{abstract}
Technologies can be promoters of breastfeeding. The scope of this review is therefore to identify health technologies and their contribution to the promotion of breastfeeding. A search was conducted in the following databases: Lilacs, SCOPUS, Medline and CINAHL, using the key words "breastfeeding," "technology" and "health promotion." The final sample consisted of 24 original articles, among which 19 technologies were identified and classified in managerial, educational and healthcare groupings. Educational technologies were predominant (12) and presented greater contributions to the promotion of breastfeeding. The combination of different technologies has revealed positive results in maintaining breastfeeding. The lack of studies with the use of managerial technologies during the lactation process, as well as the development of other research that prove the effects of each technology on increasing the prevalence of breastfeeding, was clearly detected in the review.
\end{abstract}

Key words Breastfeeding, Technology, Health promotion
Resumo As tecnologias podem ser promotoras da amamentação. Assim, o objetivo desta revisão é identificar as tecnologias em saúde e suas contribuições para a promoção do aleitamento materno. As bases de dados para busca foram: Lilacs, Scopus, Medline e CINAHL, utilizando-se os descritores "aleitamento materno", "tecnologia" e "promoção da saúde". A amostra final foi constituída por 24 artigos originais, entre eles foram identificadas 19 tecnologias classificadas em gerenciais, educacionais e assistenciais. As tecnologias educacionais predominaram (12) e apresentaram maiores contribuições para a promoção do aleitamento materno. A combinação entre diferentes tecnologias revelou resultados positivos à manutenção da amamentação. Identifica-se a carência de estudos com uso das tecnologias gerenciais durante o processo da lactação, além do desenvolvimento de outras pesquisas que comprovem os efeitos de cada tecnologia no aumento da prevalência do aleitamento materno.

Palavras-chave Aleitamento materno, Tecnologia, Promoção da saúde 


\section{Introdução}

A última pesquisa sobre a prevalência de aleitamento materno, nas capitais brasileiras e Distrito Federal, em 2008, revelou que a duração mediana do aleitamento materno exclusivo e do período total de amamentação foi 54,1 e 341,6 dias, respectivamente $^{1}$. Esses resultados confrontam as recomendações preconizadas pela Organização Mundial de Saúde - amamentação exclusiva até o sexto mês de vida e manutenção do aleitamento materno até dois anos, ou mais, com introdução de alimentos saudáveis -, apesar das campanhas e ações dos órgãos governamentais e não governamentais de incentivo ao aleitamento materno, em todo território nacional ${ }^{1,2}$.

As evidências científicas comprovam que o aleitamento materno proporciona vários benefícios, entre eles, cita-se: proteção à saúde da mulher e da criança; diminuição da morbimortalidade infantil associada à desnutrição e obesidade; contribuição com a qualidade do meio ambiente ao reduzir a produção de resíduos; cooperação com a economia financeira da família e do Estado; e melhoria da saúde da sociedade. Porém, é notório afirmar, que amamentar é uma prática complexa uma vez que a mulher e a sua família podem receber estímulos desencadeadores ao desmame precoce, tais como: influências das crenças e mitos; pouco ou limitado apoio dos serviços de saúde; condutas impróprias pelos fabricantes e distribuidores de fórmulas infantis; falta de proteção e incentivo ao aleitamento materno no ambiente laboral ${ }^{3}$.

No sentido de contribuir com a prática da amamentação, ações de educação em saúde por meio do diálogo, entre os usuários e profissionais, com auxílio de tecnologias, podem possibilitar a construção do conhecimento coletivo, desde que os saberes e a realidade desse público sejam respeitados, podendo dessa maneira, haver mudança de atitude ${ }^{4}$. Assim, as tecnologias são ferramentas/ações que podem trazer contribuições para a promoção do aleitamento, entre elas: ampliação do acesso a informações sobre saúde ${ }^{5,6}$, apoio às mães nas questões relativas ao aleitamento materno ${ }^{6}$ e aumento das taxas e do tempo de duração da prática de aleitar ${ }^{7}$.

As tecnologias são produtos ou processos que permitem o envolvimento dos profissionais na prestação do cuidado ao usuário e no desenvolvimento do processo de educação em saúde, contribuindo para o fornecimento de informações relevantes ao público-alvo ${ }^{8}$. Na promoção do aleitamento materno, as ações de educação em saúde exigem engajamento e comprometimento da equipe de saúde envolvida na assistência à mulher e a sua família. Por outro lado, o apoio/ acolhimento/orientação por parte dos profissionais da saúde pode ser determinante para o início e continuidade da amamentação9

As tecnologias em saúde são resultados provenientes de conhecimentos científicos para a produção de bens materiais, ou não, utilizadas durante a intervenção em situação prática do dia a dia e/ou no âmbito da pesquisa, buscando a resolução de problemas humanos e estruturais relacionados à saúde ${ }^{10,11}$. Elas podem também ultrapassar outras dimensões, permitindo aos profissionais repensar no processo de trabalho, sua dinamicidade, no intuito de melhorar a qualidade do serviço prestado ao usuário ${ }^{12}$. Por isso, uma concepção mais abrangente de tecnologia considera que o vínculo entre usuário-trabalhador é um trabalho vivo, um processo de relações humanas que resulta a corresponsabilização dos atores envolvidos na produção do cuidado em saúde, tendo como fim a solução de um problema. Assim, os momentos de fala e escuta na prestação desse cuidado devem respeitar a autonomia e incorporar os saberes dos usuários de saúde ${ }^{13}$.

Os pesquisadores da saúde classificam as tecnologias em: 1) gerenciais - conjunto de ações teórico-práticas para administrar as ações e serviços de saúde, cujo objetivo é intervir nas práticas profissionais com a finalidade de melhorar a sua qualidade (manuais, rotinas institucionais, acolhimento e vínculo); 2) educacionais - conjunto sistemático de conhecimento científico que permite planejar, executar, controlar e acompanhar o processo educacional formal ou informal, e, assim, favorecer a construção e reconstrução do conhecimento (cartilhas, folhetos, vídeos); 3) tecnologias assistenciais (TA) - conjunto de saberes técnico-científicos sistematizados, processuais e instrumentais, o qual possibilita a promoção da qualidade da assistência à saúde ao cliente (teorias e escalas) $8,10,14,15$.

A equipe envolvida na assistência à saúde pode prestar o cuidado à mulher e à sua família e/ou desenvolver atividades educativas promotoras do aleitamento materno por meio dessas tecnologias. Portanto, a identificação do tipo de tecnologias e suas contribuições podem auxiliar a redirecionar as práticas educativas e de cuidado vigentes voltadas para a saúde materno-infantil. Ademais, podem indicar lacunas no conhecimento quanto ao tipo de tecnologia ou pesquisa empregada e resultados referentes à temática. Nessa perspectiva, o objetivo deste estudo foi identificar 
as tecnologias em saúde e suas contribuições para a promoção do aleitamento materno.

\section{Método}

Estudo de revisão integrativa da literatura, que permite a identificação e a síntese de várias publicações, e possibilita por meio da identificação de lacunas no conhecimento, a análise mais específica de determinado fenômeno e o rápido acesso aos resultados de pesquisas para auxiliar, mediante o saber crítico, na tomada de decisão no cenário do cuidado ${ }^{16,17}$.

O percurso metodológico desta revisão integrativa foi alicerçado nas seguintes etapas: 1) identificação da questão norteadora, estabelecimento dos critérios de inclusão e exclusão dos artigos, busca nas bases de dados para identificação dos estudos; 2) categorização dos estudos e extração dos dados; 3) avaliação dos estudos incluídos na revisão; 4) discussão e interpretação dos resultados; 5) síntese das informações evidenciadas nos artigos ${ }^{18,19}$. A questão norteadora desse estudo foi a seguinte: quais as tecnologias em saúde utilizadas ou desenvolvidas pela equipe multidisciplinar e suas contribuições para a promoção do aleitamento materno?

Os critérios de inclusão estabelecidos foram: artigos originais que respondessem a questão norteadora dessa revisão, disponíveis na íntegra, publicados em português, inglês ou espanhol, entre os anos de 2004 e 2014. Esta delimitação temporal levou em consideração a criação, em 2004, da Rede de Tecnologias Sociais que objetivou mobilizar a sociedade para discutir, de maneira inclusiva, sobre tecnologias capazes de promover a melhoria da qualidade de vida das pessoas ${ }^{11}$. As teses, dissertações, monografias, editoriais, artigos de revisão (narrativa, sistemática e integrativa), resumos de eventos, relatos de caso ou de experiência foram excluídos desta pesquisa.

A busca dos artigos nas bases de dados, Literatura Latino-Americana e do Caribe em Ciên- cias da Saúde (Lilacs), Scopus, Medical Literature Analysis and Retrieval System Online (Medline) e Cumulative Index to Nursing and Allied Health Literature (CINAHL), ocorreu em julho de 2014, a partir da associação dos Descritores em Ciências da Saúde (DeCS) e Medical Subject Headin$g s(\mathrm{MeSH})$ por meio do operador booleano and: aleitamento materno/breast feeding; promoção da saúde/health promotion; tecnologia/ technology.

O número de publicações encontrado, nesta revisão, foi determinado pelo cruzamento entre os três descritores controlados, exceto na base de dados LILACS por esta não ter originado nenhum resultado. Nesta base, optou-se por associar os descritores aos pares (Tabela 1).

Como resultado dos cruzamentos dos descritores, 1.675 publicações foram identificadas. Em seguida, foram lidos todos os títulos, e por não haver palavras ou ideias correlacionadas à temática do estudo foram excluídas 1037 publicações, restando 638 para análise dos resumos. A partir dessa análise, 49 artigos foram pré-selecionados para a leitura do texto completo. Após a verificação criteriosa, em resposta a questão norteadora dessa revisão e aos critérios de inclusão estabelecidos, 29 artigos foram submetidos à análise do rigor metodológico, segundo os critérios do Critical Appraisal Skills Programme (CASP) ${ }^{20}$, a partir da qual 24 artigos foram classificados na categoria A. Portanto, a amostra final foi composta por 24 artigos originais (Figura 1).

Alguns desses artigos selecionados estavam indexados em mais de uma base de dados e, por esse motivo, foram contabilizados uma vez conforme a primeira base de dados selecionada, de acordo com a ordem de busca: Lilacs, Scopus, Medline e CINAHL.

Para a extração dos dados, adaptou-se um instrumento validado no Brasil ${ }^{21}$ com os seguintes itens: autores, ano, bases de dados, periódico, país, características metodológicas dos artigos e resultados alcançados.

$\mathrm{Na}$ avaliação do rigor metodológico das publicações, utilizou-se um instrumento adaptado

Tabela 1. Publicações sobre tecnologias desenvolvidas ou utilizadas para a promoção do aleitamento materno entre 2004 e 2014.

\begin{tabular}{lcccrr}
\hline \multicolumn{1}{c}{ Cruzamentos } & LILACS & SCOPUS & MEDLINE & CINAHL & TOTAL \\
\hline Aleitamento materno AND tecnologia & 06 & -- & -- & -- & 06 \\
Aleitamento materno AND promoção da saúde & 100 & -- & -- & -- & 100 \\
$\begin{array}{l}\text { Aleitamento materno AND promoção da saúde } \\
\text { AND tecnologia }\end{array}$ & -- & 667 & 589 & 313 & 1569 \\
Amostra & 106 & 667 & 589 & 313 & 1675 \\
\hline
\end{tabular}




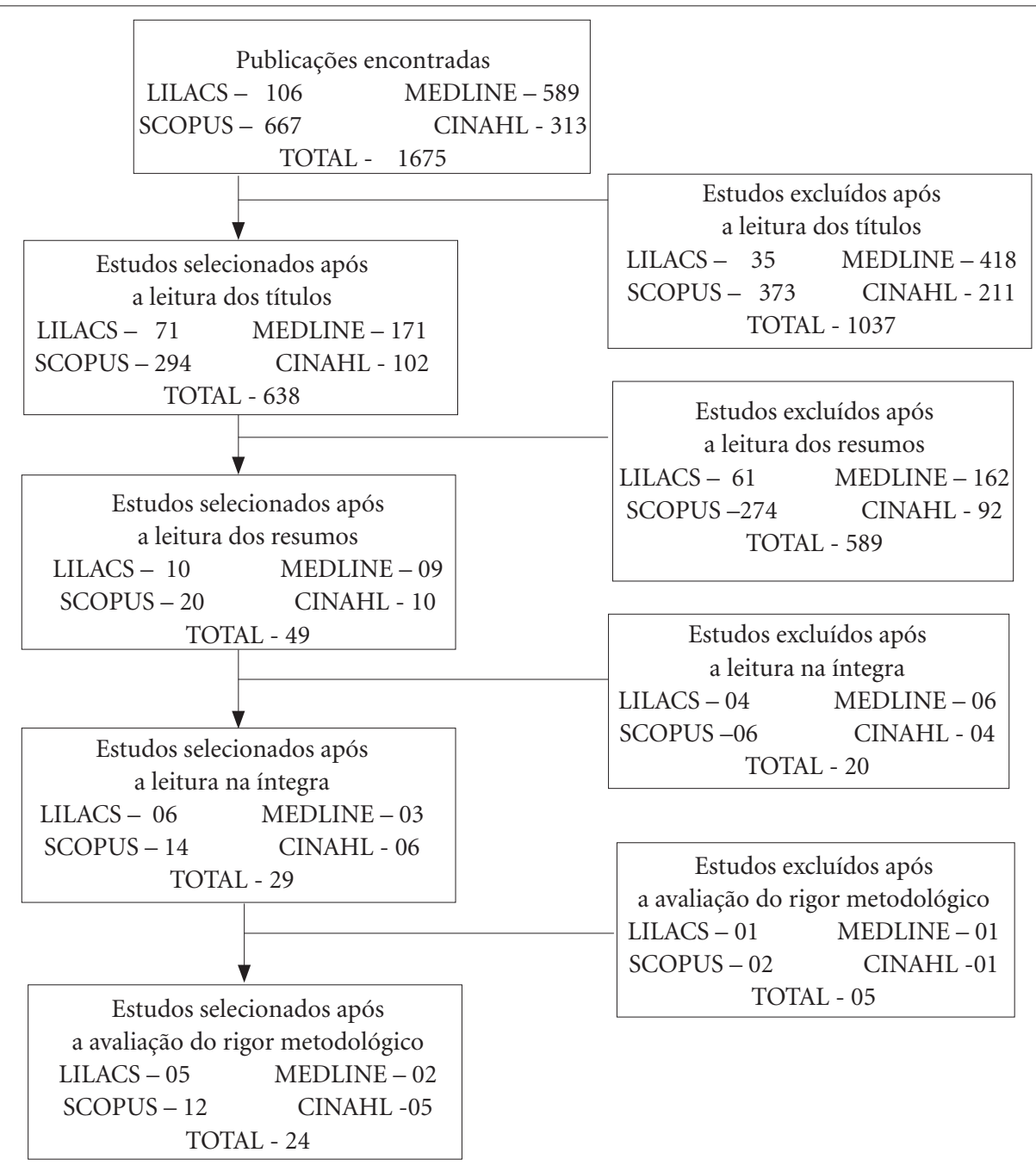

Figura 1. Descrição das etapas de seleção dos artigos incluídos na revisão integrativa.

do CASP $^{20}$ composto por dez itens a partir dos quais os estudos são classificados em duas categorias - A e B - de acordo com a pontuação obtida. Aqueles que conseguem entre 10 e seis pontos pertencem à categoria $\mathrm{A}$ - estudos de boa qualidade metodológica e viés reduzido; os da categoria B - estudos com qualidade metodológica satisfatória, mas com potencial de viés aumentado - alcançam o mínimo de cinco pontos. Quanto ao nível de evidência, as publicações foram avaliadas segundo o conceito proposto pela Prática Baseada em Evidências, classificadas em sete níveis ${ }^{22}$.

A fim de facilitar a visualização e compreensão das informações registradas nos artigos, uma síntese será apresentada em um quadro como também a classificação das tecnologias, presentes nos artigos, será resumida em uma figura. Essa classificação das tecnologias ${ }^{10}$ - gerenciais, educacionais e assistenciais- direcionará a discussão e a interpretação dos resultados.

\section{Resultados}

Dos 24 artigos $^{23-46}$ incluídos na revisão integrativa (Quadro 1), 12 encontravam-se na base de dados SCOPUS ${ }^{28-31} ; 21$ haviam sido publicados nos últimos cinco anos ${ }^{24-27,21-41,43-46}$; oito em periódicos dos $\mathrm{EUA}^{29,33-35,39,43,44,46}$ e do Reino Uni$\mathrm{do}^{28,30-32,36-38,42}$. Quanto à área de atuação dos pesquisadores, havia 13 publicações ${ }^{25-28,32,35-39,42,44,45}$ 
na enfermagem; onze $23,29,33,36,39,41,45,46$ na medicina; três ${ }^{24,39,43}$ na psicologia; e duas em cada uma das seguintes áreas: fonoaudiologia e odontologia $^{24,40}$, nutrição ${ }^{24,34}$ e informática ${ }^{35,37}$.

Em relação à classificação hierárquica metodológica proposta pela Prática Baseada em Evidências em sete níveis ${ }^{22}$ foram identificados apenas quatro níveis: cinco artigos ${ }^{23,32,35,37,46}$ alcançaram o nível 2 (ensaio clínico randomizado); seis $^{24,28,30,38-40}$ o nível 3 (ensaio clínico sem randomização); seis ${ }^{31,33,34,36,44,45}$ o nível 4 (estudos de coorte ou caso-controle)e sete ${ }^{25-27,29,40,41,43}$ o nível 6 (estudo descritivo ou qualitativo).

A maioria dos estudos apresentou mais de uma tecnologia para a promoção do aleitamento materno (Quadro 1). Essas tecnologias descritas nos artigos contemplaram todas as classificações (gerenciais, educacionais e assistenciais) ${ }^{10}$ adota- das nesta revisão integrativa. Entre as 19 tecnologias encontradas neste estudo, merece destaque o fato de que 12 foram classificadas em educacionais (Figura 2).

\section{Discussão}

Frente aos desafios - pessoais, sociais, culturais presentes no cotidiano da prática do aleitamento materno, a equipe de saúde prestadora do cuidado à mulher durante o período gravídico-puerperal, tem desenvolvido ou utilizado tecnologias para a promoção dessa prática. Estes recursos são considerados práticos, de fácil aplicação e com inúmeros benefícios à saúde da mulher e da criança ${ }^{15}$. Podem também contribuir para o

Quadro 1. Síntese das informações evidenciadas nos artigos selecionados da revisão integrativa, entre os anos de 2004 e 2014 , de acordo com autores, ano e país de publicação, base de dados, objetivo, tipo de estudo, tecnologia e contribuições para a promoção do aleitamento materno.

\begin{tabular}{|c|c|c|c|c|}
\hline $\begin{array}{l}\text { Autores /Ano } \\
\text { País da } \\
\text { publicação } \\
\text { Base de dados }\end{array}$ & Objetivo & $\begin{array}{l}\text { Tipo de } \\
\text { estudo }\end{array}$ & Tecnologia & $\begin{array}{c}\text { Contribuições } \\
\text { para a promoção } \\
\text { do aleitamento } \\
\text { materno (AM) }\end{array}$ \\
\hline $\begin{array}{l}\text { Junior, Martinez }{ }^{23} \\
2007 \\
\text { Brasil } \\
\text { LILACS }\end{array}$ & $\begin{array}{l}\text { Avaliar o impacto de um modelo de incentivo } \\
\text { ao aleitamento materno baseado no apoio e } \\
\text { orientação de mães de recém-nascidos pré- } \\
\text { termo nas taxas de aleitamento materno nos } \\
\text { primeiros seis meses após a alta hospitalar. }\end{array}$ & $\begin{array}{l}\text { Ensaio } \\
\text { clínico } \\
\text { randomizado }\end{array}$ & $\begin{array}{l}\text { Aconselhamento; } \\
\text { vínculo profissional e } \\
\text { usuário }\end{array}$ & $\begin{array}{l}\text { Aumento das taxas } \\
\text { de AM }\end{array}$ \\
\hline $\begin{array}{l}\text { Brasileiro et al. }{ }^{24} \\
2010 \\
\text { Brasil } \\
\text { LILACS }\end{array}$ & $\begin{array}{l}\text { Investigar se mães trabalhadoras formais, } \\
\text { participantes de um programa de incentivo ao } \\
\text { aleitamento materno, mantêm a amamentação } \\
\text { por mais tempo do que mães que não têm esse } \\
\text { apoio após o retorno ao trabalho. }\end{array}$ & $\begin{array}{l}\text { Quase- } \\
\text { experimental }\end{array}$ & $\begin{array}{l}\text { Aconselhamento; } \\
\text { vínculo profissional e } \\
\text { usuário }\end{array}$ & $\begin{array}{l}\text { Aumento das taxas } \\
\text { de AM }\end{array}$ \\
\hline $\begin{array}{l}\text { Linhares et al. }{ }^{25} \\
2013 \\
\text { Brasil } \\
\text { LILACS } \\
\end{array}$ & $\begin{array}{l}\text { Identificar as estratégias para promoção do } \\
\text { aleitamento materno em mulheres grávidas, as } \\
\text { mulheres que amamentam e os atores da rede de } \\
\text { apoio social para o processo de amamentação. }\end{array}$ & $\begin{array}{l}\text { Pesquisa- } \\
\text { ação }\end{array}$ & $\begin{array}{l}\text { Vínculo profissional e } \\
\text { usuário }\end{array}$ & $\begin{array}{l}\text { Discussão sobre a } \\
\text { prática do AM }\end{array}$ \\
\hline $\begin{array}{l}\text { Oliveira, Pagliuca }^{26} \\
2013 \\
\text { Brasil } \\
\text { LILACS }\end{array}$ & $\begin{array}{l}\text { Descrever o processo de avaliação de tecnologia } \\
\text { educativa, com relação aos aspectos de conteúdo } \\
\text { da literatura de cordel sobre amamentação. }\end{array}$ & Descritivo & Literatura de cordel & $\begin{array}{l}\text { Avaliação de } \\
\text { tecnologia }\end{array}$ \\
\hline $\begin{array}{l}\text { Rodrigues et al. }{ }^{27} \\
2013 \\
\text { Brasil } \\
\text { LILACS } \\
\end{array}$ & $\begin{array}{l}\text { Validar o conteúdo e aparência do álbum } \\
\text { seriado "Eu posso amamentar meu filho" } \\
\text { junto às puérperas internadas no alojamento } \\
\text { conjunto. }\end{array}$ & Descritivo & Álbum seriado & $\begin{array}{l}\text { Avaliação de } \\
\text { tecnologia }\end{array}$ \\
\hline $\begin{array}{l}\text { Hauck et al. }{ }^{28} \\
2007 \\
\text { Reino Unido } \\
\text { SCOPUS }\end{array}$ & $\begin{array}{l}\text { Avaliar os efeitos de um jornal sobre a } \\
\text { prevalência do aleitamento materno, e as } \\
\text { percepções de conselhos conflitantes, auto- } \\
\text { gestão e auto-eficácia para aleitamento materno } \\
\text { desde o nascimento até } 12 \text { semanas após o parto. }\end{array}$ & $\begin{array}{l}\text { Quase- } \\
\text { experimental }\end{array}$ & $\begin{array}{l}\text { Jornal; } \\
\text { aconselhamento; } \\
\text { Breastfeeding Self- } \\
\text { Efficacy Scale-Short } \\
\text { Form (BSES-SF) } \\
\end{array}$ & $\begin{array}{l}\text { Nenhum efeito } \\
\text { sobre a duração ou } \\
\text { taxa de AM }\end{array}$ \\
\hline
\end{tabular}


Quadro 1. Síntese das informações evidenciadas nos artigos selecionados da revisão integrativa, entre os anos de 2004 e 2014 , de acordo com autores, ano e país de publicação, base de dados, objetivo, tipo de estudo, tecnologia e contribuições para a promoção do aleitamento materno.

\begin{tabular}{|c|c|c|c|c|}
\hline $\begin{array}{l}\text { Autores /Ano } \\
\text { País da } \\
\text { publicação } \\
\text { Base de dados }\end{array}$ & Objetivo & $\begin{array}{l}\text { Tipo de } \\
\text { estudo }\end{array}$ & Tecnologia & $\begin{array}{l}\text { Contribuições } \\
\text { para a promoção } \\
\text { do aleitamento } \\
\text { materno (AM) }\end{array}$ \\
\hline $\begin{array}{l}\text { Whelan, Kearney }{ }^{29} \\
2010 \\
\text { EUA } \\
\text { SCOPUS }\end{array}$ & $\begin{array}{l}\text { Descrever uma avaliação do teste piloto de uma } \\
\text { produção de teatro-fórum para a sensibilização } \\
\text { da amamentação. }\end{array}$ & Descritivo & Teatro-fórum & $\begin{array}{l}\text { Discussão sobre a } \\
\text { prática do AM }\end{array}$ \\
\hline $\begin{array}{l}\text { Labarère et al. } \\
2011 \\
\text { Reino Unido } \\
\text { SCOPUS }\end{array}$ & $\begin{array}{l}\text { Avaliar a eficácia de uma intervenção baseada } \\
\text { em CD-ROM para o aumento das taxas de } \\
\text { aleitamento materno. }\end{array}$ & $\begin{array}{l}\text { Quase- } \\
\text { experimental }\end{array}$ & CD-ROM & $\begin{array}{l}\text { Nenhum efeito } \\
\text { sobre a duração ou } \\
\text { taxa de AM }\end{array}$ \\
\hline $\begin{array}{l}\text { Pannu et al. }{ }^{31} \\
2011 \\
\text { Reino Unido } \\
\text { SCOPUS }\end{array}$ & $\begin{array}{l}\text { Determinar o efeito de mães que receberam } \\
\text { material de promoção da saúde e de educação } \\
\text { pré-natal e / ou pós-natal sobre os resultados da } \\
\text { amamentação em Perth, Austrália Ocidental. }\end{array}$ & Coorte & $\begin{array}{l}\text { Vídeo; } \\
\text { aconselhamento; } \\
\text { folheto }\end{array}$ & $\begin{array}{l}\text { Aumento das taxas } \\
\text { de AM }\end{array}$ \\
\hline $\begin{array}{l}\text { Kronborg et al. }{ }^{32} \\
2012 \\
\text { Reino Unido } \\
\text { SCOPUS }\end{array}$ & $\begin{array}{l}\text { Avaliar o efeito de um programa de treinamento } \\
\text { pré-natal para o conhecimento, auto-eficácia } \\
\text { e os problemas relacionados ao aleitamento } \\
\text { materno e sua duração. }\end{array}$ & $\begin{array}{l}\text { Ensaio } \\
\text { clínico } \\
\text { randomizado }\end{array}$ & $\begin{array}{l}\text { Filme; } \\
\text { aconselhamento }\end{array}$ & $\begin{array}{l}\text { Nenhum efeito } \\
\text { sobre a duração ou } \\
\text { taxa de AM }\end{array}$ \\
\hline $\begin{array}{l}\text { Tornese et al. }{ }^{33} \\
2012 \\
\text { EUA } \\
\text { SCOPUS }\end{array}$ & $\begin{array}{l}\text { Analisar a relação entre o escore LATCH } \\
\text { avaliado nas primeiras } 24 \text { horas de vida e após } \\
\text { o parto e identificar um ponto de corte para } \\
\text { o escore, a fim de identificar as mulheres com } \\
\text { maior risco de aleitamento não exclusivo que } \\
\text { podem precisar de apoio adicional para a } \\
\text { amamentação. }\end{array}$ & Coorte & $\begin{array}{l}\text { Método LATCH } \\
\text { (Latches, Audible, Type, } \\
\text { Comfort, } \\
\text { Help) }\end{array}$ & $\begin{array}{l}\text { Detecção de } \\
\text { necessidade de apoio } \\
\text { adicional para a } \\
\text { prática do AM. }\end{array}$ \\
\hline $\begin{array}{l}\text { Witt et al. } \\
2012 \\
\text { EUA } \\
\text { SCOPUS }\end{array}$ & $\begin{array}{l}\text { Avaliar o impacto da intervenção de um } \\
\text { consultor de lactação pós-parto em uma } \\
\text { prática pediátrica para melhorar o apoio à } \\
\text { amamentação. }\end{array}$ & $\begin{array}{l}\text { Caso- } \\
\text { Controle }\end{array}$ & Aconselhamento & $\begin{array}{l}\text { Aumento das taxas } \\
\text { de AM; Redução } \\
\text { da introdução de } \\
\text { outros alimentos à } \\
\text { dieta do bebê. }\end{array}$ \\
\hline $\begin{array}{l}\text { Edwards et al. }{ }^{35} \\
2013 \\
\text { EUA } \\
\text { SCOPUS }\end{array}$ & $\begin{array}{l}\text { Desenvolver e avaliar um agente de computador } \\
\text { interativo em animação projetado para fornecer } \\
\text { informações e apoio a amamentação às mães } \\
\text { interessadas em amamentar. }\end{array}$ & $\begin{array}{l}\text { Ensaio } \\
\text { clínico } \\
\text { randomizado }\end{array}$ & $\begin{array}{l}\text { Computador } \\
\text { interativo; Iowa Infant } \\
\text { Feeding Attitude Scale } \\
\text { (IIFAS); Breastfeeding } \\
\text { Self-Efficacy Scale- } \\
\text { Short Form(BSES-SF) }\end{array}$ & $\begin{array}{l}\text { Nenhum efeito } \\
\text { sobre a duração ou } \\
\text { taxa de AM }\end{array}$ \\
\hline $\begin{array}{l}\text { Donnan et al. }{ }^{36} \\
2013 \\
\text { Reino Unido } \\
\text { SCOPUS }\end{array}$ & $\begin{array}{l}\text { Obter modelos de previsão tanto para a } \\
\text { iniciação e interrupção do aleitamento materno } \\
\text { utilizando variáveis demográficas, psicológicas e } \\
\text { obstétricas. }\end{array}$ & Coorte & $\begin{array}{l}\text { Iowa Infant Feeding } \\
\text { Attitude Scale } \\
\text { (IIFAS); teoria do } \\
\text { comportamento } \\
\text { planejado }\end{array}$ & $\begin{array}{l}\text { Detecção de } \\
\text { necessidade de apoio } \\
\text { adicional para a } \\
\text { prática do AM. }\end{array}$ \\
\hline $\begin{array}{l}\text { Giles et al. }{ }^{37} \\
2014 \\
\text { Reino Unido } \\
\text { SCOPUS }\end{array}$ & $\begin{array}{l}\text { Avaliar a eficácia de uma intervenção em meio } \\
\text { escolar projetado para melhorar as motivações } \\
\text { dos jovens para amamentar. }\end{array}$ & $\begin{array}{l}\text { Ensaio } \\
\text { clínico } \\
\text { randomizado }\end{array}$ & $\begin{array}{l}\text { Aconselhamento; } \\
\text { teoria do } \\
\text { comportamento } \\
\text { planejado } \\
\text { (questionário) }\end{array}$ & $\begin{array}{l}\text { Aumento de } \\
\text { intenções e atitudes } \\
\text { positivas para } \\
\text { amamentar; redução } \\
\text { da introdução de } \\
\text { outros alimentos à } \\
\text { dieta do bebê. }\end{array}$ \\
\hline
\end{tabular}

continua

aumento de conhecimento, mudança de atitude e adoção de comportamentos mais saudáveis como preconizado pelas ações de promoção da saúde ${ }^{47,48}$. Portanto, as tecnologias não são apenas equipamentos e artefatos, mas também constituem os saberes que permitem construí-las a 
Quadro 1. Síntese das informações evidenciadas nos artigos selecionados da revisão integrativa, entre os anos de 2004 e 2014 , de acordo com autores, ano e país de publicação, base de dados, objetivo, tipo de estudo, tecnologia e contribuições para a promoção do aleitamento materno.

\begin{tabular}{|c|c|c|c|c|}
\hline $\begin{array}{l}\text { Autores /Ano } \\
\text { País da } \\
\text { publicação } \\
\text { Base de dados }\end{array}$ & Objetivo & $\begin{array}{l}\text { Tipo de } \\
\text { estudo }\end{array}$ & Tecnologia & $\begin{array}{l}\text { Contribuições } \\
\text { para a promoção } \\
\text { do aleitamento } \\
\text { materno }(\mathrm{AM})\end{array}$ \\
\hline $\begin{array}{l}\text { Hannula et al. } .^{38} \\
2014 \\
\text { Reino Unido } \\
\text { SCOPUS }\end{array}$ & $\begin{array}{l}\text { 1. Avaliar o impacto do apoio } \\
\text { intensificado para a amamentação durante o } \\
\text { período perinatal. }\end{array}$ & $\begin{array}{l}\text { Quase- } \\
\text { experimental }\end{array}$ & $\begin{array}{l}\text { Aconselhamento; } \\
\text { vínculo profissional } \\
\text { e usuário; website; } \\
\text { Método LATCH } \\
\text { (Latches, Audible, } \\
\text { Type, Comfort, Help); } \\
\text { Iowa Infant Feeding } \\
\text { Attitude Scale (IIFAS) } \\
\text { e Breastfeeding Self- } \\
\text { Efficacy Scale-Short } \\
\text { Form (BSES-SF) }\end{array}$ & $\begin{array}{l}\text { Aumento das taxas } \\
\text { de AM }\end{array}$ \\
\hline $\begin{array}{l}\text { Jiang et al. }{ }^{39} \\
2014 \\
\text { EUA } \\
\text { SCOPUS }\end{array}$ & $\begin{array}{l}\text { Avaliar o efeito de uma intervenção por } \\
\text { mensagens de texto no celular sobre as práticas } \\
\text { de alimentação infantil. }\end{array}$ & $\begin{array}{l}\text { Quase- } \\
\text { experimental }\end{array}$ & $\begin{array}{l}\text { Mensagens de texto no } \\
\text { celular }\end{array}$ & $\begin{array}{l}\text { Aumento das taxas } \\
\text { de AM; redução } \\
\text { da introdução de } \\
\text { outros alimentos à } \\
\text { dieta do bebê. } \\
\end{array}$ \\
\hline $\begin{array}{l}\text { Corrêa et al. }{ }^{40} \\
2013 \\
\text { Brasil } \\
\text { MEDLINE }\end{array}$ & $\begin{array}{l}\text { Descrever o desenvolvimento e a avaliação } \\
\text { do conteúdo do site referente às funçôes orais } \\
\text { apresentados no "Portal babies". }\end{array}$ & Descritivo & Website & $\begin{array}{l}\text { Desenvolvimento } \\
\text { e avaliação de } \\
\text { tecnologia }\end{array}$ \\
\hline $\begin{array}{l}\text { Charkazi et al. }{ }^{41} \\
2013 \\
\text { Índia } \\
\text { MEDLINE }\end{array}$ & $\begin{array}{l}\text { Investigar a situação de aleitamento materno } \\
\text { nos dois primeiros anos de vida dos bebês e os } \\
\text { fatores que influenciaram através do modelo } \\
\text { BASNEF (behavior, attitudes, subjetive norms } \\
\text { and enabling factores). }\end{array}$ & Descritivo & $\begin{array}{l}\text { Modelo } \\
\text { comportamental } \\
\text { BASNEF } \\
\text { (questionário) }\end{array}$ & $\begin{array}{l}\text { Atitudes positivas } \\
\text { para amamentar }\end{array}$ \\
\hline $\begin{array}{l}\text { Lin et al. }{ }^{42} \\
2008 \\
\text { Reino Unido } \\
\text { CINAHL }\end{array}$ & $\begin{array}{l}2 . \quad \text { Analisar a efetividade de um } \\
\text { programa de educação pré-natal em aleitamento } \\
\text { para primíparas que escolheram parto cesariano } \\
\text { eletivo; avaliar a efetividade do encorajamento } \\
\text { para práticas positivas para aleitamento em } \\
\text { alojamento conjunto e para aumentar o AME no } \\
\text { hospital e um mês após o parto. }\end{array}$ & $\begin{array}{l}\text { Quase- } \\
\text { experimental }\end{array}$ & $\begin{array}{l}\text { Vídeo; folheto; escala } \\
\text { de atitudes }\end{array}$ & $\begin{array}{l}\text { Aumento das taxas } \\
\text { de AM; aumento de } \\
\text { atitudes positivas } \\
\text { para amamentar. }\end{array}$ \\
\hline $\begin{array}{l}\text { Dyson et } \text { al. }^{43} \\
2010 \\
\text { EUA } \\
\text { CINAHL }\end{array}$ & $\begin{array}{l}\text { Analisar fatores psicológicos que influenciam a } \\
\text { intenção de amamentar entre adolescentes que } \\
\text { esperam o primeiro bebê e que vivem em áreas } \\
\text { urbanas desfavorecidas na Inglaterra. }\end{array}$ & Descritivo & $\begin{array}{l}\text { Teoria do } \\
\text { comportamento } \\
\text { planejado } \\
\text { (questionário) }\end{array}$ & $\begin{array}{l}\text { Discussão sobre a } \\
\text { prática do AM }\end{array}$ \\
\hline $\begin{array}{l}\text { Ho, McGranth } \\
2011 \\
\text { EUA } \\
\text { CINAHL }\end{array}$ & $\begin{array}{l}\text { Explorar atitudes de mães tailandesas e variáveis } \\
\text { sociodemográficas para continuação do } \\
\text { aleitamento em seis semanas de pós-parto. }\end{array}$ & Coorte & $\begin{array}{l}\text { Iowa Infant Feeding } \\
\text { Attitude Scale (IIFAS) }\end{array}$ & \begin{tabular}{|l} 
Detecção de \\
necessidade de \\
apoio adicional para \\
a prática do AM; \\
discussão sobre a \\
prática do AM. \\
\end{tabular} \\
\hline $\begin{array}{l}\text { Dai et al. }^{45} \\
2013 \\
\text { Austrália } \\
\text { CINAHL }\end{array}$ & $\begin{array}{l}\text { Avaliar a confiabilidade e validade de uma } \\
\text { versão chinesa da IIFAS entre as mulheres pós- } \\
\text { parto. }\end{array}$ & Coorte & $\begin{array}{l}\text { Iowa Infant Feeding } \\
\text { Attitude Scale (IIFAS) }\end{array}$ & $\begin{array}{l}\text { Detecção de } \\
\text { necessidade de apoio } \\
\text { adicional para a } \\
\text { prática do AM }\end{array}$ \\
\hline $\begin{array}{l}\text { Bonuck et al. }{ }^{46} \\
2014 \\
\text { EUA } \\
\text { CINAHL }\end{array}$ & $\begin{array}{l}\text { Determinar a afetividade de cuidado primário } \\
\text { de intervenções pré e pós-natal para aumentar o } \\
\text { aleitamento materno. }\end{array}$ & $\begin{array}{l}\text { Ensaio } \\
\text { clínico } \\
\text { randomizado }\end{array}$ & $\begin{array}{l}\text { Aconselhamento; } \\
\text { vínculo profissional e } \\
\text { usuário }\end{array}$ & $\begin{array}{l}\text { Aumento das taxas } \\
\text { de AM }\end{array}$ \\
\hline
\end{tabular}




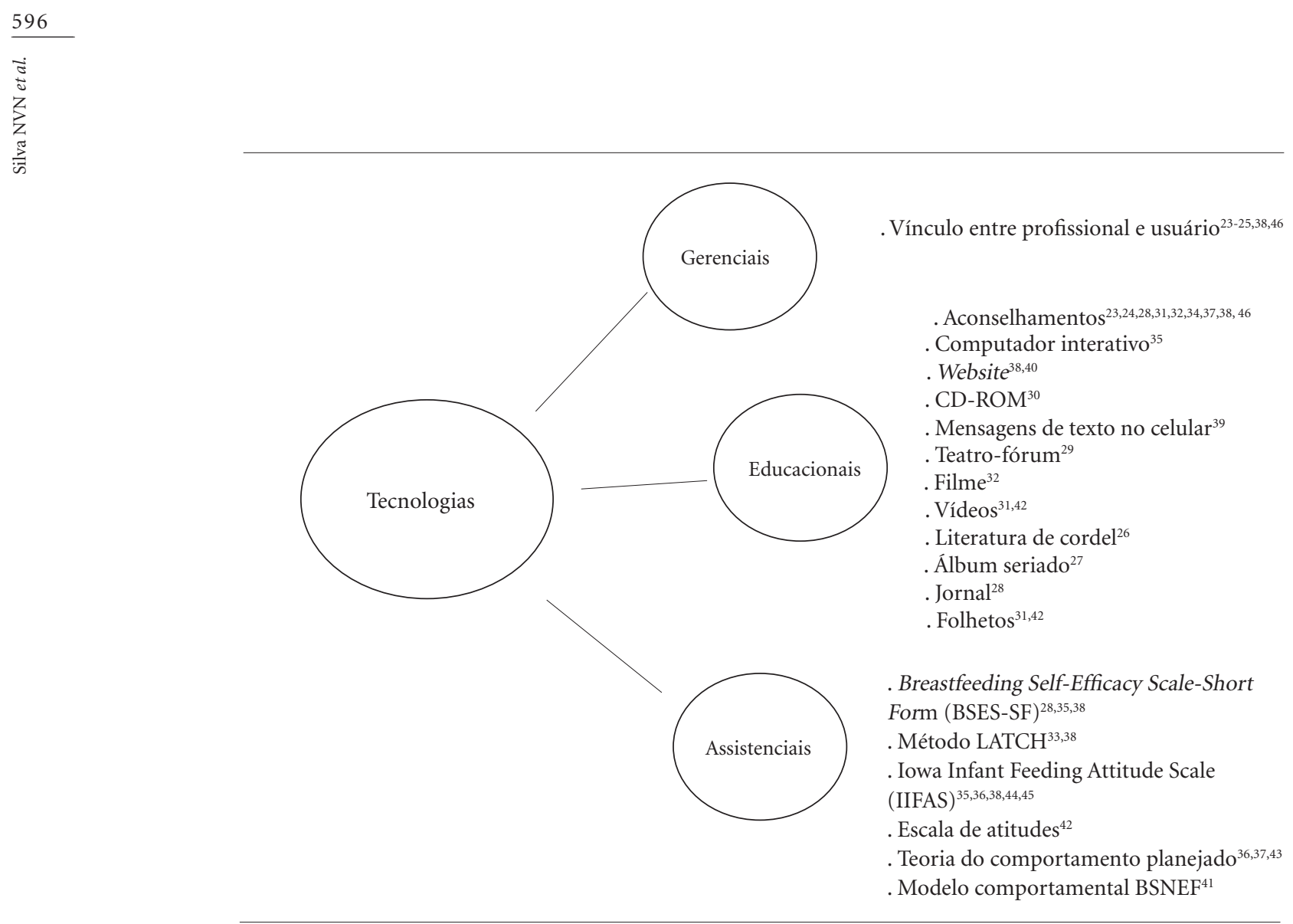

Figura 2. Categorização das publicações incluídas na revisão integrativa, segundo classificação e tecnologia utilizada ou desenvolvida para a promoção do aleitamento materno no período de 2004 a 2014. Recife, PE, Brasil, 2015.

Fonte: Adaptado de Nietsche et al. ${ }^{10}$.

partir das relações humanas com finalidades preestabelecidas pelo processo de trabalho ${ }^{12}$.

A utilização das tecnologias referentes às relações sociais presentes nos processos gerenciais como o acolhimento, o vínculo, a autonomização e a responsabilização - podem intervir na produção do cuidado ${ }^{12}$. Essas tecnologias são provenientes das relações humanas com intuito de satisfazer as necessidades dos usuários e valorizar os envolvidos (trabalhador e usuário de saúde) com vistas ao fortalecimento da concretização do cuidado $^{14}$. Entre as tecnologias gerenciais (TG) citadas, a única identificada nesta revisão foi o vínculo ${ }^{23-25,38,46}$

O vínculo como tecnologia, deve ser promovido não apenas entre o usuário e o profissional da saúde, mas entre a mãe e o seu filho para solidificar a competência parental ${ }^{23}$. Em especial, entre recém-nascidos prematuros, o vínculo precoce entre mãe e filho ${ }^{23}$ faz parte das boas práticas, garantido em todas as unidades neonatais pela Portaria MS/GM no 390, de 03 de setembro de 2012, a qual permite aos pais a permanência integral durante a internação do filho, o que tem promovido o aumento nas taxas de aleitamento materno.

Nesta revisão constatou-se que o vínculo entre o profissional e o usuário ${ }^{23-25,38,46}$ e o aconselhamento sobre amamentação $0^{23,24,31,34,38,46}$ são tecnologias que quando aplicadas proporcionam maiores taxas de aleitamento materno. No entanto, ressalta-se que essas tecnologias, em grande parte, foram desenvolvidas em associação com outras tecnologias por meio de estudos não experimentais, por isso não se pode afirmar a mais eficaz na promoção da amamentação.

Ainda em relação a essas tecnologias - vínculo e aconselhamento -, apenas dois artigos ${ }^{23,46}$ resultaram de ensaios clínicos randomizados, tipo de estudo que avalia os efeitos de uma intervenção. Tal fato sinaliza a necessidade de desenvolvimento de estudos experimentais bem delineados com a aplicação de tecnologias promotoras do aleitamento materno. 
Nesta revisão percebeu-se que as tecnologias educacionais (TE) foram as mais utilizadas e desenvolvidas pelos profissionais da saúde, a saber: aconselhamentos $23,24,28,31,32,34,37,38,46$, computador interativo ${ }^{35}$, website ${ }^{38,40}, \mathrm{CD}-\mathrm{ROM}^{30}$, mensagens de texto no celular ${ }^{39}$, teatro-fórum ${ }^{29}$, filme ${ }^{32}$, ví$\operatorname{deos}^{31,42}$, literatura de cordel $^{26}$, álbum seriado ${ }^{27}$, jornal $^{28} \mathrm{e}$ folheto ${ }^{31,42}$.

As tecnologias, fundamentadas na perspectiva crítica, criativa e transformadora, são instrumentos usados por educadores para facilitar a formação do conhecimento e proporcionar a participação de todos no processo educativo, em prol do desenvolvimento da autonomia e da construção da cidadania dos envolvidos ${ }^{49}$.

No âmbito da saúde, o compartilhamento de experiências, entre os profissionais e os pais, pode ser promovido em grupos de apoio, por meio do acolhimento e aconselhamento, a fim de reforçar a competência parental e estreitamento dos vínculos entre pais e filhos recém-nascidos em favor do aleitamento materno ${ }^{25,50}$. O apoio e a educação em saúde sobre amamentação quando desenvolvidos em conjunto com a tecnologia de aconselhamento individual e em grupo pode impactar no aumento das taxas de amamentação exclusiva $^{51}$.

Contudo, destaca-se que aconselhar vai além de fornecer informações. Significa comunicar-se de maneira simples e empática, colocando-se o usuário no centro das atenções ${ }^{52}$. O aconselhamento pode ser difundido por meio do diálogo pedagógico entre profissionais e os usuários. $\mathrm{O}$ diálogo por sua vez é essencial para resolução dos problemas de saúde por promover o compartilhamento de saberes populares com os dos especialistas ${ }^{4}$. Portanto, o diálogo e escuta ativa do profissional deve fazer com que as mulheres sintam-se acolhidas e apoiadas nos aspectos referentes à amamentação ${ }^{52}$.

O trabalho formal da mulher mãe tem sido apresentado como umas das causas do desmame precoce $^{53}$, contudo o apoio e o aconselhamento no ambiente laboral, mediante atividades teóricas e práticas em aleitamento materno podem contribuir na manutenção da amamentação exclusiva até o sexto mês de vida da criança. Assim, considerando-se que a licença maternidade ainda é de 120 dias na maioria das instituições brasileiras, programas de incentivo ao aleitamento no ambiente de trabalho durante o retorno das atividades das mulheres podem elevar as taxas de aleitamento materno exclusivo até os seis meses ${ }^{24}$.

Entre as tecnologias educacionais ressalta-se que as tecnologias da informação e comunica- ção (TIC) são recursos promissores e têm sido largamente desenvolvidas e aplicadas na área da saúde, como a mensagem de texto no celular ${ }^{39}$, o computador interativo ${ }^{35}$, o website $^{38,40}$ e o $\mathrm{CD}$ $-\mathrm{ROM}^{30}$. Essas tecnologias identificadas nos artigos selecionados trazem contribuições positivas à promoção do aleitamento materno e estão relacionadas ao aumento nas suas $\operatorname{taxas}^{38,39}$, à redução da introdução de outros alimentos à dieta do bebê, antes do sexto mês de vida ${ }^{39} \mathrm{e}$ a estudo de desenvolvimento e avaliação de tecnologias na temática ${ }^{40}$.

A inserção das tecnologias da informação e comunicação no ensino possibilita que o indivíduo busque diferentes fontes de informação, as quais podem ajudar na resolução dos problemas da vida e do trabalho $0^{54}$. Assim, a crescente incorporação de recursos tecnológicos, a partir das necessidades educacionais da sociedade, pode proporcionar aquisição de informações sobre aleitamento materno, promovendo a autonomia dos indivíduos ${ }^{6}$. E para o usuário usufruir desse aprendizado é preciso avaliações contínuas de usabilidade desses ambientes no sentido de permitir o fácil acesso e a compreensão das tecnologias propostas ${ }^{48}$.

As tecnologias educativas também podem ser lúdicas, como o teatro-fórum ${ }^{29}$, o filme ${ }^{32}$, o vídeo ${ }^{31,42}$ e a literatura de corde ${ }^{26}$. Evidências científicas de estudos experimentais comprovam a eficácia do uso de atividades lúdicas para promoção da saúde, estimulando a compreensão de determinados assuntos de forma prazerosa e reflexiva ${ }^{55}$. Contudo, entre as tecnologias lúdicas identificadas, apenas o vídeo ${ }^{31}$ esteve associado ao aumento da taxa de aleitamento materno, enquanto o teatro-fórum ${ }^{29}$ proporcionou a discussão de aspectos de sua prática.

A tecnologia do teatro-fórum é uma maneira de compartilhar histórias de vida entre os atores e espectadores a partir de um problema social, antimodelo. O público é questionado e convidado a participar da atuação na tentativa de amenizar ou superar a opressão encenada ${ }^{56}$. Permite ainda a discussão, o debate e o ensaio da realidade, além de promover a consciência crítica quanto aos aspectos envolvidos no aleitamento materno por meio do controle das situações propostas ${ }^{29}$.

O filme como atividade lúdica, construído a partir de opiniões dos pais sobre aleitamento materno, foi aplicado, no Irã, como um método de treinamento popular sobre esse tema ${ }^{57}$. Esse princípio empregado na construção desse filme é fundamental no desenvolvimento de tecnologias em atenção ao atendimento das necessidades 
educacionais do público-alvo ${ }^{6}$. Por sua vez, os vídeos educativos proporcionam a aquisição de informações em saúde como recurso auxiliar, pois são de fácil acesso e permitem uma compreensão e reprodução das informações ${ }^{58}$. Entretanto, a aplicação de maneira isolada, dessas tecnologias citadas - o filme e o vídeo - deve ser avaliada, pois se verificou que não contribuíram para a manutenção da amamentação exclusiva ${ }^{57}$.

Um cordel validado sobre amamentação, para as pessoas com deficiências visuais, disponibilizado em duas modalidades, áudio e impresso, além de reforçar a importância do vínculo entre mãe e filho durante o aleitamento materno, esclarece mitos e tabus sobre essa prática ${ }^{26}$. Isso indica que as tecnologias voltadas ao público com deficiências podem garantir o direito de acesso às informações de saúde e minimizar os riscos de adoecimento ${ }^{59}$.

Algumas tecnologias educativas desenvolvidas no formato impresso - álbum seriado ${ }^{27}$, jornal ${ }^{28} \mathrm{e}$ folheto $\mathrm{o}^{31,42}$ - são relevantes por potencializarem a aquisição de informações, visto que há uma carência de cuidados básicos de saúde entre os usuários $^{60}$. A informação nesse formato está centrada na linguagem escrita e visual, aspectos importantes a serem avaliados após o desenvolvimento das tecnologias, pois o conteúdo e as ilustrações devem ser claros e de fácil compreensão, características fundamentais das tecnologias educacionais ${ }^{27}$.

Os folhetos ${ }^{31,42}$, quando aplicados com outras tecnologias, vídeo e aconselhamento, contribuíram no aumento das taxas da prática do aleitar. Tal resultado não foi evidenciado quando a utilização do jornal ${ }^{28}$ foi associada à tecnologia do aconselhamento. No entanto, outros estudos experimentais necessitam serem desenvolvidos para a confirmação desses resultados.

Ainda em relação ao uso do jornal, pode-se equacionar que os resultados obtidos variam de acordo como as informações foram transmitidas às mulheres e familiares. Muitas vezes, essas informações são fornecidas de forma impositiva e vertical, sem levar em consideração os saberes e as limitações da mulher e dos membros da sua rede social ${ }^{9}$. Esse aspecto realça a importância do profissional da saúde, ser um facilitador do processo educativo, mediante o diálogo e a escuta ativa, entre pais e familiares sobre assuntos pertinentes a saúde da criança, com a utilização ou não de tecnologias ${ }^{61}$.

O enfermeiro, nesse contexto, foi o profissional mais envolvido com as pesquisas desta revisão e tem relevante função de mediador das ações de educação em saúde, visto que é o profissional que atua em diversos âmbitos da assistência à saúde - na atenção primária, secundária ou terciária $^{62}$. A atuação desse profissional está ancorada nos princípios de educação em saúde que evidencia uma prática participativa, dialogada e reflexiva entre os usuários e equipe de saúde. Contudo, na sua prática profissional ainda são identificadas posturas autoritárias, transmissão vertical do conhecimento, negação da subjetividade dos usuários e reprodução do modelo biomédico. Essa postura deve ser repensada por contrariar as propostas da promoção da saúde, pautadas na decisão compartilhada entre usuário e profissional ${ }^{63}$.

As tecnologias em saúde também empregam elementos centrados em saberes estruturados no processo de trabalho ${ }^{12}$, como as tecnologias assistenciais (TA), provenientes de investigações, aplicações de teorias e experiências profissionais ${ }^{10}$, tais quais as selecionadas nesta revisão: Breastfeeding Self-Efficacy Scale - Short Form (BSES-SF) ${ }^{28,35,38}$, método LATCH (Latches, Audible, Type, Comfort, Help) ${ }^{33,38}$, Iowa Infant Feeding

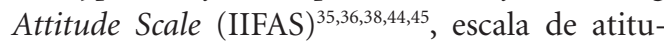
$\operatorname{des}^{42}$, teoria do comportamento planejado ${ }^{36,37,43}$ e o modelo teórico comportamental BASNEF ${ }^{41}$. Essa modalidade de tecnologia apresenta demanda crescente quando comparadas com revisão similar voltada para o aleitamento materno ${ }^{15}$.

A BSES-SF, conhecida como escala de autoeficácia para a amamentação, é composta por 14 itens baseados nos pressupostos da teoria de autoeficácia de Bandura de 1977. Essa teoria identifica o que as pessoas pensam, sentem e como são motivadas diante de situações que necessitam de persistência para enfrentar uma experiência negativa $^{27}$. Assim a escala BSES-SF permite identificar por meio dos escores obtidos se a mulher encontra-se disposta a manter o aleitamento materno exclusivo ou apresenta comportamentos favoráveis ao desmame precoce ${ }^{64}$. Nesse sentido, conforme alguns artigos analisado ${ }^{28,35,38}, \mathrm{o}$ aumento da prevalência do aleitamento materno foi associado à autoeficácia, o que reforça a ideia de que a motivação interfere na prática da amamentação.

O método LATCH é uma maneira sistemática de documentar informações sobre a amamentação observada, formado por cinco itens que se referem à pega, à deglutição audível, ao tipo de mamilo, ao conforto da mãe e à necessidade de ela ter ajuda para levar o bebê ao peito ${ }^{65}$. Para cada item, os escores variam de zero a dois e a pontuação total do método varia de zero a dez. Considera-se uma boa avaliação quando os escores situam-se entre oito e dez ${ }^{66}$, pois isso indica 
que houve associação com a manutenção do aleitamento materno exclusivo. Porém, se os escores forem baixos, infere-se que há a necessidade de essa mulher, ainda durante a internação até a alta hospitalar, contar com o apoio adicional a respeito dos aspectos pertinentes à lactação ${ }^{33}$.

Já a IIFAS composta por 17 itens, permite mensurar, a partir de elementos da teoria do comportamento planejado, atitudes das mulheres referentes à preferência pelo aleitamento materno ou pelo uso de fórmulas lácteas ${ }^{67}$. A alta pontuação dessa escala, entre 17 e 85 pontos, está associada ao aumento da prevalência de aleitamento materno. Assim, as escalas de atitudes podem ser instrumentos eficientes para determinar a necessidade de apoio adicional à nutriz para o aleitamento materno ${ }^{36,44,45}$, o aumento de duração dessa prática e as atitudes positivas para a continuidade da amamentação ${ }^{38,42}$.

Questionários construídos a partir da teoria do comportamento planejado e aplicados em pesquisas $^{36,37,43}$, consideram que o comportamento final do indivíduo depende da sua intenção de comportamento. Essa teoria considera os seguintes aspectos: as atitudes (fatores individuais), normas subjetivas (pressões sociais) e controle percebido (grau de facilidade ou dificuldade para executar tal comportamento $)^{68}$. Isso pode ser útil para formular e avaliar intervenções de mudança de comportamento ${ }^{37}$; prever fatores que interferem na prática da amamentação; e detectar a necessidade de apoio adicional durante a vivência do aleitamento materno ${ }^{36}$.

Em um dos artigos, verificou-se que os pesquisadores construíram um questionário a partir do modelo comportamental BASNEF, alicerçado em pressupostos da teoria do comportamento planejado. Esse modelo permite afirmar que o conhecimento das nutrizes sobre o aleitamento materno, e o apoio do marido e de sua mãe durante essa prática colaboram para o desenvolvimento de atitudes positivas referentes à adesão à amamentação. Por isso, esses comportamentos devem ser considerados nos programas de incentivo ao aleitamento ${ }^{41}$.

\section{Considerações finais}

Nesta revisão viu-se que as tecnologias em saúde mais predominante foram as tecnologias educacionais e na maioria dos casos em que foram aplicadas, contribuíram para a manutenção do aleitamento materno. Por sua vez, observou-se que as tecnologias assistenciais apresentaram uma demanda crescente e auxiliaram na detecção de apoio adicional à mulher para amamentar, principalmente por meio de escalas. Já as tecnologias gerenciais foram pouco aplicadas e, quando associadas a outras tecnologias, resultaram em efeitos positivos na promoção da amamentação. Assim, essas tecnologias, por contemplarem a dimensão das relações humanas, precisam ser mais exploradas nas pesquisas e na assistência à mulher durante o processo da amamentação, pois esta prática é permeada pela dinâmica relacional entre o usuário de saúde e os profissionais envolvidos na assistência.

Resultados favoráveis também foram verificados à manutenção do aleitamento materno quando houve associação entre tecnologias. Entretanto, foram poucos os estudos que comprovaram os efeitos dessas associações direcionadas ao aumento da prevalência e da duração da amamentação.

Ainda observou-se que o Enfermeiro tem tido um papel relevante na utilização dessas tecnologias para o alcance de melhores taxas de aleitamento materno. Tal fato permite afirma-se que é desafiante e ao mesmo tempo estimulante a aplicação de tecnologias por uma equipe multidisciplinar capaz de promover efeitos significativos nos indicadores da prática de aleitar.

Em síntese, os resultados dessa revisão integrativa revelaram a necessidade de desenvolvimento de outros estudos, com diferentes métodos de pesquisa, relacionados ao uso de tecnologias - educacionais, assistenciais e gerenciais - na promoção do aleitamento materno, podendo contribuir, dessa maneira, na melhoria da qualidade da assistência prestada à mulher/ nutriz e sua família.

\section{Colaboradores}

Os autores NVN Silva e NFC Sousa participaram de todas as fases de construção do artigo, desde a pesquisa no banco de dados a organização do texto completo e as autoras MGL Vasconcelos e CM Pontes contribuíram na fase inicial da pesquisa nas bases de dados, nas definições do método e revisão da redação final. 


\section{Referências}

1. Brasil. Ministério da Saúde (MS). II Pesquisa de Prevalência de Aleitamento Materno nas Capitais Brasileiras e Distrito Federal. Brasília: MS; 2009.

2. Brasil. Ministério da Saúde (MS). Iniciativa Hospital Amigo da Criança. Brasília: MS; 2011.

3. Organização Pan-Americana da Saúde. Semana Mundial de Aleitamento Materno, 1-7 agosto 2014. Amamentação: uma questão contemporânea em um mundo globalizado [Internet]. 2014. [acessado 15 Jan 2017] Disponível em: www.pahoorg/bra/image/storie/.../ brief\%20report\%202014\%20portugues.pdf

4. Vasconcelos EM, Vasconcelos MOD, Silva MO. A contribuição da educação popular para a reorientação das práticas e da política de saúde no Brasil. Rev FAEEBA - Educação e Contemporaneidade 2015; 24(43):89-106.

5. Vasconcellos-Silva PR, Castiel LD. As novas tecnologias de autocuidado e os riscos do autodiagnóstico pela internet. Rev Panam Salud Publica 2009; 26(2):172-175.

6. Vasconcelos MGL, Góes FSN, Fonseca LMM, Ribeiro LM, Scochi CGS. Avaliação de um ambiente digital de aprendizagem pelo usuário. Acta Paul Enferm 2013; 26(1):36-41.

7. Flax VL, Negerie M, Ibrahim AU, Leatherman S, Daza EJ, Bentley ME. Integrating Group Counseling, cell phone messaging, and participant-generated songs and dramas into a Microcredit Program Increases Nigerian Women's Adherence to internacional breastfeeding recommendations. J Nutr 2014; 144(7):11201124.

8. Nietsche EA, Teixeira E, Medeiros HP. Tecnologias cuidativo-educacionais: uma possibilidade para o emponderamento do/a enfermeiro/a? Porto Alegre: Moriá; 2014.

9. Monte GCSB, Leal LP, Pontes CM. Rede social de apoio à mulher na amamentação. Cogitare Enferm 2013; 18(1):148-155.

10. Nietsche EA, Backes VMS, Colomé CLM, Ceratti RN, Ferraz F. Tecnologias educacionais, assistenciais e gerenciais: uma reflexão a partir da concepção de docentes de enfermagem. Rev Latino Am Enferm 2005; 13(3):344-353.

11. Lorenzetti J, Trindade LL, Pires DEP, Ramos FRS. Tecnologia, inovação tecnológica e saúde: uma reflexão necessária. Texto contexto Enferm 2012; 21(2):432439.

12. Merhy EE. Em busca de ferramentas analisadoras das Tecnologias em Saúde: a informação e o dia a dia de um serviço, interrogando e gerindo trabalho em saúde. In: Merhy EE, Onoko R, organizadores. Agir em saúde: um desafio para o público. $3^{\text {a }}$ ed. São Paulo: Hucitec; 2007. p. 113-160.

13. Merhy EE. A perda da dimensão cuidadora na produção da saúde um discussão do modelo assistencial e da intervenção no seu modo de trabalhar a assistência. In: Sistema Único de Saúde em Belo Horizonte - Reescrevendo o público. São Paulo: Xamã; 1998. p. 103-120. Parte II.
14. Rossi FR, Lima MADL. Acolhimento: tecnologia leve nos processos gerenciais do enfermeiro. Rev Bras Enferm 2005; 58(3):305-310.

15. Joventino ES, Dodt RCM, Araujo TL, Cardoso MVLML, Silva VM, Ximenes LB. Tecnologias de enfermagem para promoção do aleitamento materno: revisão integrativa da literatura. Rev Gaúcha Enferm 2011; 32(1):176-184

16. Botelho LLR, Cunha CCA, Macedo M. O método da revisão integrativa nos estudos organizacionais. Gestão e Sociedade 2011; 5(11):121-136.

17. Brevidelli MM, Sertório SCM. Trabalho de Conclusão de Curso - Guia Prático para Docentes e Alunos da Área da Saúde. 4a ed. Iatria: Erica; 2010.

18. Mendes KDS, Silveira RCCP, Galvão CM. Revisão integrativa: método de pesquisa para a incorporação de evidências na saúde e na enfermagem. Texto Contexto Enferm 2008; 17(4):758-764.

19. Lopes CMM, Galvão CM. Posicionamento cirúrgico: evidências para o cuidado de enfermagem. Rev Latino Am Enferm 2010; 18(2):155-162.

20. Critical Appraisal Skills Programme (CASP) 2014 CASP Checklists (URL used). Oxford. CASP [internet]. 2014 [acessado 2014 Jul 10] Disponível em: http://www.casp-uk.net/checklists

21. Ursi ES, Galvão CM. Prevenção de lesões de pele no período perioperatório: revisão integrativa da literatura. Rev Latino Am Enferm 2006; 14(1):124-131.

22. Galvão CM. Editorial. Níveis de evidência. Acta Paul Enferm 2006; 19(2).

23. Junior WS, Martinez FE. Effect of intervention on the rates of breastfeeding of very low birth weight newborns. J Pediatr 2007; 83(6):541-546.

24. Brasileiro AA, Possobon RF, Carrascoza KC, Ambrosano GMB, Moraes ABA. Impacto do incentivo ao aleitamento materno entre mulheres trabalhadoras formais. Cad Saúde Pública 2010; 26(9):1705-1713.

25. Linhares FMP, Pontes CM, Osório MM. Breastfeeding promotion and support strategies based on Paulo Freire's epistemological categories. Rev Nutr 2013; 26(2):125-134

26. Oliveira PMP, Pagliuca LMF. Avaliação de tecnologia educativa na modalidade de literatura de cordel sobre amamentação. Rev Esc Enferm USP 2013; 47(1):205212.

27. Rodrigues AP, Nascimento LA, Dodt RCM, Oriá MOB, Ximenes LB. Validação de um álbum seriado para promoção da autoeficácia em amamentar. Acta Paul Enferm 2013; 26(6):586-593.

28. Hauck Y, Hall WA, Jones C. Prevalence, self-efficacy and perceptions of conflicting advice and self-management: effects of a breastfeeding journal. J Adv Nurs 2007; 57(3):306-317.

29. Whelan B, Kearney JM. Promotion breastfeeding throught drama: a preliminary study. Food Sci Nutr 2010; 40(13):330-339.

30. Labarère J, Gelbert-Baudino N, Laborde L, Arragain D, Schelstraete C, François P. CD-ROM-based program for breastfeeding mothers. Matern Child Nutr 2011; 7(3):263-272. 
31. Pannu PK, Giglia RC, Binns CW, Scott JA, Oddy WH The effectiveness of health promotion materials and activities on breastfeeding outcomes. Acta Paediatr 2011; 100(4):534-537.

32. Kronborg H, Maimburg RD, Væth M. Antenatal training to improve breast feeding: a randomised trial. Midwifery 2012; 28(6):784-790.

33. Tornese G, Ronfani L, Pavan C, Demarini S, Monasta L, Davanzo R. Does the LATCH Score Assessed in the First 24 Hours After Delivery Predict Non-Exclusive Breastfeeding at Hospital Discharge? Breastfeeding Med 2012; 7(6):423-430.

34. Witt AM, Smith S, Mason MJ, Flocke SA. Integrating Routine Lactation Consultant Support into a Pediatric Practice. Breastfedding Med 2012; 7(1):38-42.

35. Edwards RA, Bickmore T, Jenkins L, Foley M, Manjourides J. Use of an interactive Computer agent to support breastfeeding. Matern Child Health J 2013; 17(10):1961-1968.

36. Donnan PT, Dalzell J, Symon A, Rauchhaus P, Monteith-Hodge E, Kellett G, Wyatt JC, Whitford HM. Prediction of initiation and cessation of breastfeeding from late pregnancy to 16 weeks: the Feeding Your Baby (FYB) cohort study. BMJ Open 2013; 3:e003274.

37. Giles M, McClenahan C, Armour C, Millar S, Rae G, Mallett J, Stewart-Knox B. Evaluation of a theory of planned behaviour-based breastfeeding intervention in Northern Irish schools using a randomized cluster design. J Health Psychol 2014; 19(1):16-35.

38. Hannula LS, Kaunonen ME, Puukka PJ. A study to promote breast feeding in the Helsinki Metropolitan area in Finland. Midwifery 2014; 30(6):696-704.

39. Jiang H, Li M, Wen LM, Hu Q, Yang D, He G, Baur LA, Dibley MJ, Qian X. Effect of Short Message Service on Infant Feeding Practice: Findings From a Community-Based Study in Shanghai, China. JAMA Pediatrics 2014; 168(5):471-478.

40. Corrêa CC, Pauleto ARC, Ferrari DV, Berretin-Felix G. Website Babies Portal: development and evaluation of the contents regarding orofacial functions. $J$ appl Oral Sci 2013; 21(6):581-589.

41. Charkazi A, Miraeiz SZ, Razzaghnejad A, Shahnazi H, Hasanzadeh A, Badleh MT. Breastfeeding status during the first two years of infants' life and its risk factors based on BASNEF model structures in Isfahan. J Edu Health Promot 2013; 2:9.

42. Lin AH, Kuo SC, Lin KC, Chang TY. Evaluating effects of a prenatal breastfeeding education programme on women with caesarean delivery in Taiwan. JCN 2008; 17(21):2838-2845

43. Dyson L, Green JM, Renfrew MJ, McMillan B, Woolridge M. Factors influencing the infant feeding decision for socioeconomically deprived pregnant teenagers: the moral dimension. Birth 2010; 37(2):141149.

44. Ho YJ, McGranth JM. Predicting breastfeeding duration related to maternal attitudes in a Taiwanese sample. JPE 2011; 20(4):188-199.
45. Dai HX, Guan XD, Li XM, You LM, Lau Y. Psychometric properties of a mainland Chinese version of the Iowa Infant Feeding Attitude Scale among postpartum women in China. Contemp Nurse 2013; 44(1):1120.

46. Bonuck K, Stuebe A, Barnett J, Labbok MH, Fletcher J, Bernstein PS. Effest of primary care intervention on breastfeeding duration and intensity. Am J Public Health 2014; 104(Supl. 1):119-127.

47. Joshi A, Wilhelm, Aguirre T, Trout K, Amadi C. An Interactive, Bilingual Touch Screen Program to Promote Breastfeeding Among Hispanic Rural Women: Usability Study. JMIR Res Protoc 2013; 2(2):1-19.

48. Góes FS, Fonseca LMM, Furtado MCC, Leite AM, Scochi CGS. Evaluation of the virtual learning object "Diagnostic reasoning in nursing applied to preterm newborns. Rev Latino Am Enferm 2011; 19(4):894901.

49. Martins AKL, Nunes JM, Nóbrega MFB, Pinheiro PNC, Souza AMA, Vieira NFC, Fernandes AFC. Literatura de cordel: tecnologia de educação para saúde e enfermagem. Rev Enferm UERJ 2011; 19(2):324-329.

50. Vasconcelos MGL, Leite AM, Scochi CGS. Vivência materna como acompanhantes de recém-nascidos. Rev Bras Saúde Matern Infant 2006; 6(1):47-57.

51. Haroon S, Das JK, Salam RA, Imdad A, Bhutta ZA. Breastfeeding promotion interventions and breastfeeding practices: a systematic review. BMC Public Health 2013; 13(Supl. 3):S20.

52. Brasil. Ministério da Saúde (MS). Saúde da criança: nutrição infantil. Aleitamento materno e alimentação complementar. Brasília: MS; 2015.

53. Queluz MC, Pereira MJ, Santos CB, Leite AM, Ricco RG. Prevalence and determinants of exclusive breastfeeding in the city of Serrana, São Paulo, Brazil. Rev Esc Enferm USP 2012; 46(3):537-543.

54. Tornaghi AJC. Tecnologias na educação: ensinando e aprendendo com as TIC: guia do cursista [internet]. Brasília: Secretaria de Educação a Distância; 2010. [acessado 2014 Dez 6]. Disponível em: http://portaldoprofessor.mec.gov.br/storage/materiais/0000011620.pdf

55. Coscrato G, Pina JC, Mello DF. Utilização de atividades lúdicas na educação em saúde: uma revisão integrativa da literatura. Acta Paul Enferm 2010; 23(2):257-263.

56. Canda CN. Teatro-fórum: propósitos e procedimentos. Urdimento [internet]. 2012 [acessado 2014 Dez 18]; 02:119-128. Disponível em: http://www.ceart,udesc.br/ppgt/urdimento/2012/12_Teatro-Forum_-propositos_e_procedimentos.pdf

57. Khayyati F, Mansouri M. The effect of training movies on exclusive breastfeeding. Pak J Med Sci 2009; 25(3):434-438.

58. Moreira CB, Bernardo EBR, Catunda HLO, Aquino PS, Santos MCL, Fernandes AFC. Construção de um Vídeo Educativo sobre Detecção Precoce do Câncer de Mama. Rev Bras Cancerol 2013; 59(3):401-407. 
59. Rodrigues SCM, Damião GC. Ambiente virtual: auxílio ao atendimento de enfermagem para surdos com base no protocolo de atenção básica. Rev Esc Enferm USP 2014; 48(4):731-738.

60. Oliveira MS, Fernandes AFC, Sawada NO. Manual educativo para o auto-cuidado da mulher mastectomizada: um estudo de validação. Texto Contexto Enferm 2008; 17(1):115-123.

61. McKellar L, Pincombe J, Henderson A. 'Coming ready or not!' Preparing parents for parenthood. BJM 2009; 17(3):160-167.

62. Silva LD, Beck CLC, Dissen CM, Tavares JP, Budó MLD, Silva HS. O enfermeiro e a educação em saúde: um estudo bibliográfico. Rev enferm UFSM 2012; 2(2):412-419.

63. Cervera DPP, Parreira BDM, Goulart BF. Educação em saúde: percepção dos enfermeiros da atenção básica em Uberaba (MG). Cien Saude Colet 2011; 16 (Supl. 1):1547-1554.

64. Souza EFC, Fernandes RAQ. Breastfeeding self-efficacy: a cohort study. Acta Paulista de Enfermagem 2014; 27(5):465-470.

65. Jensen D, Wallace S, Kelsay P. LATCH: a breastfeeding Charting System and Documentation Tool. J Obstet Gynecol Neonatal Nurs 1994; 23(1):27-32.

66. León CB, Contreras RB, Sequeros EM, Ayuso MLP, Conde AIS, Hormigos CV. Validación al castellano de una escala de evaluación de la lactancia materna: el LATCH. Análisis de fiabilidad. Index Enferm 2008; 17(3):205-209.

67. De la Mora A, Russell DW, Dungy CI, Losch M, Dusdieker L. The Iowa Infant Feeding Attitude Scale: Analysis of reliability and validity. J Appl Psychol 1999; 29(11):2362-2380.

68. Chinazzo IR, Camara SG, Frantz DG. Comportamento sexual de risco em jovens: aspectos cognitivos e emocionais. Psico USF 2014; 19(1):1-12.

Artigo apresentado em 13/12/2015

Aprovado em 03/04/2017

Versão final apresentada em 05/04/2017 